\title{
Contenido de ADN y PATRÓN DE GENES RIBOSOMALES EN EL GÉNERO MONOTí́lico STETSONIA (CACTACEAE)
}

\author{
KAREN BAUK ${ }^{1}$, FEDERICO SANTIÑAQUE ${ }^{2}$, BEATRIZ LÓPEZ-CARRO² y M. L. LAS PEÑAS ${ }^{1}$
}

\begin{abstract}
Summary: DNA content and pattern of ribosomal genes in the monotypic genus Stetsonia (Cactaceae). Stetsonia coryne is a tree is tree-sized plant, with large and infundibuliform flowers $(12-15 \mathrm{~cm})$. It is native to the deserts of northwestern Argentina and Bolivia, and is the only species of the genus Stetsonia. The aim of this study was to characterize this species cytologically. For this, the nuclear DNA content, chromosome number, karyotype, banding patterns and location of ribosomal genes of the species was determined. The DNA content analysis revealed a mixture of nuclei with three peaks, $2 \mathrm{C}, 4 \mathrm{C}$ and $8 \mathrm{C}$, indicating a process of endopoliploidy on the species, being this the first report for the tribe Browningieae. It presented chromosome number of $2 n=22$, with a symmetrical karyotype of $22 m$, with the first pair with satellite. With fluorescent chromosome banding technique, two types of bands were observed: $\mathrm{CMA}^{+} /$ DAPI associated centromere and NOR associated. With FISH technique, we observed that 18-5.8-26S loci were consistent with $\mathrm{CMA}^{+} / \mathrm{DAPI} / \mathrm{NORs}$ blocks. The signal for the $5 \mathrm{~S}$ gene is located in a centromeric region (pair 10).
\end{abstract}

Key words: Stetsonia coryne, DNA content, endopolyploidy, heterochromatin, ribosomal genes.

\begin{abstract}
Resumen: Stetsonia coryne es una planta de porte arborescente y con flores grandes (12 a $15 \mathrm{~cm}$ de largo) e infundibuliformes. Es originaria de los desiertos del noroeste de Argentina y Bolivia, y es la única especie del género Stetsonia. El objetivo de este trabajo fue caracterizar citológicamente a esta especie. Para ello se determinó el contenido de ADN nuclear, el número cromosómico, cariotipo, patrones de bandeo, y localización de genes ribosómicos de la especie. El análisis de contenido de ADN reveló una mezcla de núcleos con tres picos de $2 \mathrm{C}, 4 \mathrm{C}$ y $8 \mathrm{C}$, lo que sugiere un proceso de endopoliploidía en la especie, siendo esto el primer reporte para la tribu Browningieae. Presentó número cromosómico somático de $2 n=22$. Con la técnica de bandeo cromosómico fluorescente se identificaron dos tipos de bandas. Por último, se aplicó la técnica de FISH, se observó una co-localización del locus 18-5,8-26S con la banda $\mathrm{CMA}^{+} / \mathrm{DAPI} / \mathrm{NOR}$, la señal del $5 \mathrm{~S}$ fue localizada en el par 10 en la región centromérica. Estos resultados son los primeros para la tribu.
\end{abstract}

Palabras clave: Stetsonia coryne, contenido de ADN, endopoliploidía, heterocromatina, genes ribosomales.

\section{INTRODUCCIÓN}

La subfamilia Cactoideae comprende el $80 \%$ de las especies de cactus reconocidas hasta el momento (Anderson, 2002; Hunt et al., 2006); se caracteriza por que las plantas no presentan hojas, las aréolas no poseen gloquidios; las flores son solitarias

${ }^{1}$ Instituto Multidisciplinario de Biología Vegetal, Universidad Nacional de Córdoba-CONICET, Córdoba, Argentina.

${ }^{2}$ Instituto de Investigaciones Biológicas Clemente Estable, Avenida Italia 3318. CP. 11600, Montevideo - Uruguay.

Correo electrónico: laulaspenas@yahoo.com.ar y acampanadas; los tallos son generalmente cilindroides u obcónicos con costillas (Anderson, 2001; Trevisson \& Demaio, 2006). Dentro de esta subfamilia se encuentra Stetsonia coryne (SalmDyck) Britton \& Rose, conformando un género monotípico (Kiesling et al., 2008). El estatus taxonómico del género ha sido controvertido, ya que Ritz (2007) y Hernández- Hernandez et al. (2011) lo incluyen en la filogenia dentro del clado BCT (Browningieae, Cereeae y Trichocereeae), donde las especies más relacionadas son del género Cereus. Tradicionalmente, al género se lo incluye dentro de la tribu Browningieae (Kiesling, 1996), además, 
incluye a los géneros Armatocereus Backeb., Browningia Britton \& Rose, Jasminocereus Britton \& Rose, Neoraimondia Britton \& Rose. Estos se distribuyen en América del Sur, en los Andes y las Islas Galápagos (Anderson, 2001).

Stetsonia habita en Bolivia, Paraguay y Argentina (Kiesling et al., 2008); siendo común en el Chaco seco, en ambientes de llanura y montaña, encontrándosela hasta los $1000 \mathrm{msnm}$. Se convierte en el elemento dominante en áreas perisalinas o muy secas, ya que es muy resistente a la aridez extrema (Demaio et al., 2002). Es un cactus arborescente, la flor es grande de 12 a $15 \mathrm{~cm}$ de largo, el pericarpelo con escamas, los tépalos externos son verdes y los internos blancos. A esta especie se la utiliza localmente para fabricar artesanías, como "palos de lluvia" instrumento musical, su fruto es utilizado como mordiente para que las tinturas se fijen a las lanas; su leño sirve para sustituir al corcho en aislantes térmicos, y se las cultiva para formar cercos vivos. Sus frutos son semejantes a las tunas aunque más ácidas. También es refugio de animales, como el carpintero de los cardones (Melanerpes cactorum), que nidifica en su tronco (Demaio et al., 2002).

Pese a la relevancia de este género, el conocimiento citológico de Stetsonia es casi nulo. Por ejemplo, se desconoce su valor C. La determinación del valor $\mathrm{C}$, es la cantidad de ADN de un genoma haploide no replicado de un individuo (Orhi, 1998; Bottini, 2000; Las Peñas et al., 2014). En las angiospermas el contenido de $\mathrm{ADN}$ nuclear $(\mathrm{ADNc})$ varía entre 1 y hasta 125 pg por núcleo diploide (Bennett \& Leitch, 2010). La estimación de este parámetro ha facilitado los estudios de evolución y especiación en numerosos grupos de plantas. En Cactaceae, existen algunos estudios de medición de ADN nuclear (Palomino \& Heras, 2001; Zonneveld et al., 2005; Negron- Ortiz, 2007; Segura et al., 2007; Las Peñas et al., 2013). El contenido de ADN por genoma básico $(\mathrm{Cx})$ en las especies de la subfamilia Cactoideae varía desde 0,54 a 3,46 pg (Bennett \& Leitch, 2010). Además, en la familia Cactaceae se ha observado en varias especies el proceso de endopoliploidia (Palomino \& Heras, 2001; Zonneveld et al., 2005; Negron- Ortiz, 2007; Segura et al., 2007), el cual es un proceso en el que las células se someten a ciclos repetidos de síntesis de ADN sin división celular, resultando una multiplicación exponencial del contenido de ADN nuclear (Barow, 2006; Leitch et al., 2013).

Por otra parte, los estudios citológicos muestran que el número básico propuesto para Cactaceae es $\mathrm{x}=11$, siendo la principal variación la poliploidía (Pinkava, 2002; Las Peñas, 2009). Herramientas como el bandeo cromosómico fluorescente e hibridación in situ fluorescente (FISH) permiten identificar homologías cromosómicas inter o intra específicas, y son ampliamente usadas en cariosistemática (Greilhuber, 1984). En poblaciones argentinas se han realizado estudios citogenéticos para algunos géneros de cactus (Das \& Mohanty, 2006; Las Peñas et al., 2009, 2011, 2013) pero de Stetsonia sólo se conoce el número cromosómico (2n= 22; Bauk \& Las Peñas, 2012).

Por lo tanto, el objetivo propuesto en el presente trabajo es aportar información del contenido de ADN nuclear y citogenética para el género Stetsonia, siendo los primeros para la especie.

\section{Materiales y Métodos}

Las plantas fueron recolectadas en localidades argentinas de las provincias de Santa Fe (Ruta Nacional $11 \mathrm{~km} \mathrm{734,} \mathrm{a} 20 \mathrm{~km}$ de Vera, Las Peñas et al. 73) y Catamarca (entre San Martín y Casa

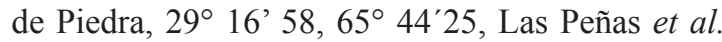
524). Los especímenes fueron plantados en macetas con una mezcla de tierra y arena en partes iguales y cultivados en la colección de Cactaceae que se encuentra en FCEFyN-UNC. Los ejemplares de herbario correspondientes fueron depositados en el herbario del Museo Botánico de Córdoba, CORD.

Para calcular el contenido de ADN nuclear se siguió la metodología de Doležel et al. (2007) con pequeñas modificaciones. En una cápsula de Petri conteniendo $0,5 \mathrm{ml}$ de la solución de Otto I (ácido cítrico $0,1 \mathrm{M}$ y $0,5 \%$ de Tween 20 ) y $0,2 \mathrm{ml}$ de $1 \%$ de PVP, se colocaron $100 \mathrm{mg}$ de plántulas obtenido de semillas germinadas de 30 días de Stetsonia coryne y $25 \mathrm{mg}$ de tejido foliar de Zea mays L. $\mathrm{CE}-777(2 \mathrm{C}=5,43 \mathrm{pg})$, utilizado como estándar interno. El material se picó finamente con hoja de afeitar y se filtró a través de una malla de nylon de $45 \mu \mathrm{m}$ para separar la suspensión de núcleos del material grueso. La suspensión de núcleos se 


\section{K. Bauk et al. - ADN y patrón de genes ribosomales en el género monotípico Stetsonia}

incubó por 10 a 60 minutos a temperatura ambiente; luego se adicionó $0,5 \mathrm{ml}$ de tampón de Otto II $(0,4$ $\mathrm{M}$ de Na2HPO4 12H2O) y se realizó un nuevo filtrado. Se agregaron $125 \mu \mathrm{l}$ de ARNasa e igual cantidad de yoduro de propidio, para su análisis en el CF (Becton-Dickinson, San José, EEUU), sintonizado para emitir luz a $488 \mathrm{~nm}$.

Se llevaron a cabo tres estimaciones de ADN para cada población (5.000 núcleos por análisis), en tres días diferentes. El valor $2 \mathrm{C}$ se calcula como pico promedio de la muestra $\mathrm{x}$ valor $2 \mathrm{C}$ del estándar (pg) / pico promedio del estándar.

Para los estudios cromosómicos se usaron raíces de 5 plantas adultas, las cuales fueron pretratadas con 8-hidroxiquinoleína al 0,002M durante 24 horas en frío y se fijaron en alcohol-ácido acético (3:1) se conservaron en freezer hasta su uso.

Las raíces fueron sometidas a digestión enzimática con Pectinex ${ }^{\circledR}$. Para ello fueron lavadas en agua destilada durante 10 minutos, y luego fueron maceradas con una solución enzimática por 40 minutos. Los meristemas fueron aplastados en un portaobjetos con una gota de ácido acético al 45\%, el cubreobjetos fue removido por congelamiento con aire líquido y se colocó los preparados en freezer hasta su uso. Para la doble tinción CMA/ DAPI y FISH, se siguieron los protocolos descriptos en Las Peñas et al. (2011). Las sondas utilizadas fueron para la detección de los genes ribosómicos 18-5,8-26S (45S) la pTa71 (Gerlach \& Bedbrook, 1979) y para el $5 S$ la sonda específica de Cactaceae (Las Peñas et al., 2011) y una sonda específica para la especie. El marcado de las sondas se realizó con biotina y dioxigenina por "nick-traslation", la detección de las mismas se realizó mediante anticuerpos conjugados con fluorocromos antibiotina con fluoresceína (FITC) y anti-digoxigenina con rodamina. Además, para las mediciones de los cromosomas los preparados fueron coloreados con Giemsa al 2\% preparada en el momento. El tiempo de coloración fue de entre 5 a 10 minutos, la intensidad de la coloración se verificó a partir de los dos minutos en un microscopio, finalmente se montó el portaobjetos con Entellan.

Por último, las preparaciones cromosómicas fueron fotografiadas utilizando el microscopio Zeiss Axiophot con una cámara digital Leica DFC300FX, con los filtros apropiados para cada técnica. El análisis de las imágenes se realizó con el programa IMAGE J (http://rsb.info.nih.gov/ij/).

\section{Resultados}

Las muestras de plantas jóvenes de Stetsonia coryne fueron analizadas junto al testigo Zea mays L. CE - 777 el cual no fue incluido en las figuras, en el gráfico se incluyó solo la especie para poder visualizar los 3 picos $(2 \mathrm{C}=3,05,4 \mathrm{C}=6,01,8 \mathrm{C}=$ $12,2)$ con lo cual podemos sugerir un proceso de endopoliploidía en la especie (Fig. 1 A).

Stetsonia coryne presentó un número cromosómico somático de $2 \mathrm{n}=22$ en las poblaciones analizadas (Fig. 1 B). Los cromosomas fueron pequeños con un largo promedio de $3,76 \mu \mathrm{m}$, y la media del genoma haploide de 41,34 $\mu \mathrm{m}$. La fórmula cariotípica fue de $11 \mathrm{~m}$, En la Tabla 1, se incluyen las características cariotípicas para cada par cromosómico que fueron usadas para generar el idiograma (Fig. 1 F). El cariotipo fue simétrico con pequeñas diferencias entre los pares cromosómicos, presentando un índice de asimetría intracromosómico $\left(\mathrm{A}_{1}\right)$ de 0,16 y un intercromosómico $\left(A_{2}\right)$ de 0,20 . Con la técnica clásica no fue posible observar satélites.

Con la técnica de CMA/DAPI se pudo observar el siguiente patrón de bandas: un par cromosómico presentó regiones ricas en $\mathrm{CMA}^{+} / \mathrm{DAPI}^{-}$asociadas a NORs y un par con una banda $\mathrm{CMA}^{+} / \mathrm{DAPI}$ asociada al centrómero (Fig. 1C, D). El porcentaje de heterocromatina CMA/DAPI fue de 3,33 \% del total de genoma haploide. Por último, se mapearon los genes ribosómicos, la señal de hibridación para el gen 18-5,8-26S fue localizada en el primer par cromosómico $m$, en la región de satélite y parte del cromosoma que lo porta. La señal para el gen $5 \mathrm{~S}$ se localizó en un par $m$ en una región pericentromérica (Fig. 1E). El tamaño, número e intensidad de las señales de ambos genes $\mathrm{ADNr}$ fueron similares entre los homólogos.

\section{Discusión}

Elanálisis del contenido de ADN nuclear mediante citometría de flujo es útil para explorar el nivel de ploidía, la detección de híbridos intraespecíficos y apomixis, la determinación del tamaño del genoma y la endorreduplicación, la identificación de sexo (Ohri, 1998; Costich et al., 1991; Bennett et al., 2000). En Cactaceae, este método se ha utilizado en especies de Pereskia, especies de Opuntioideae 
Bol. Soc. Argent. Bot. 51 (2) 2016

A
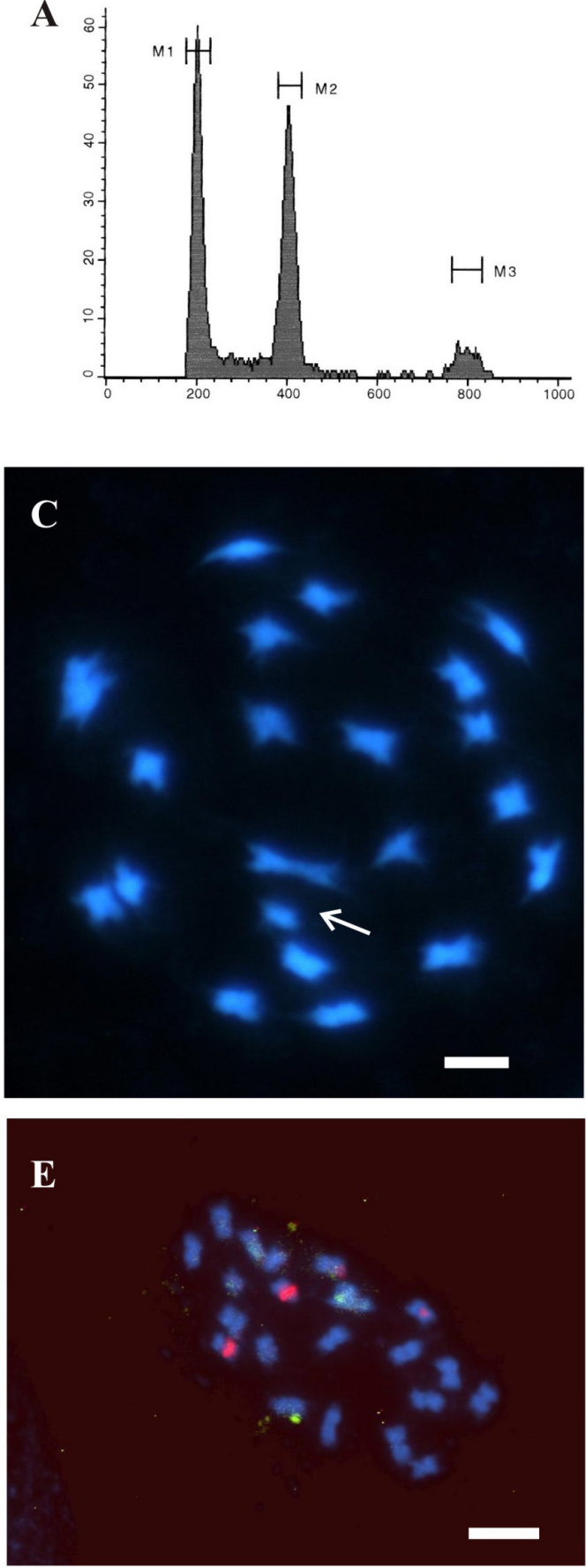

B
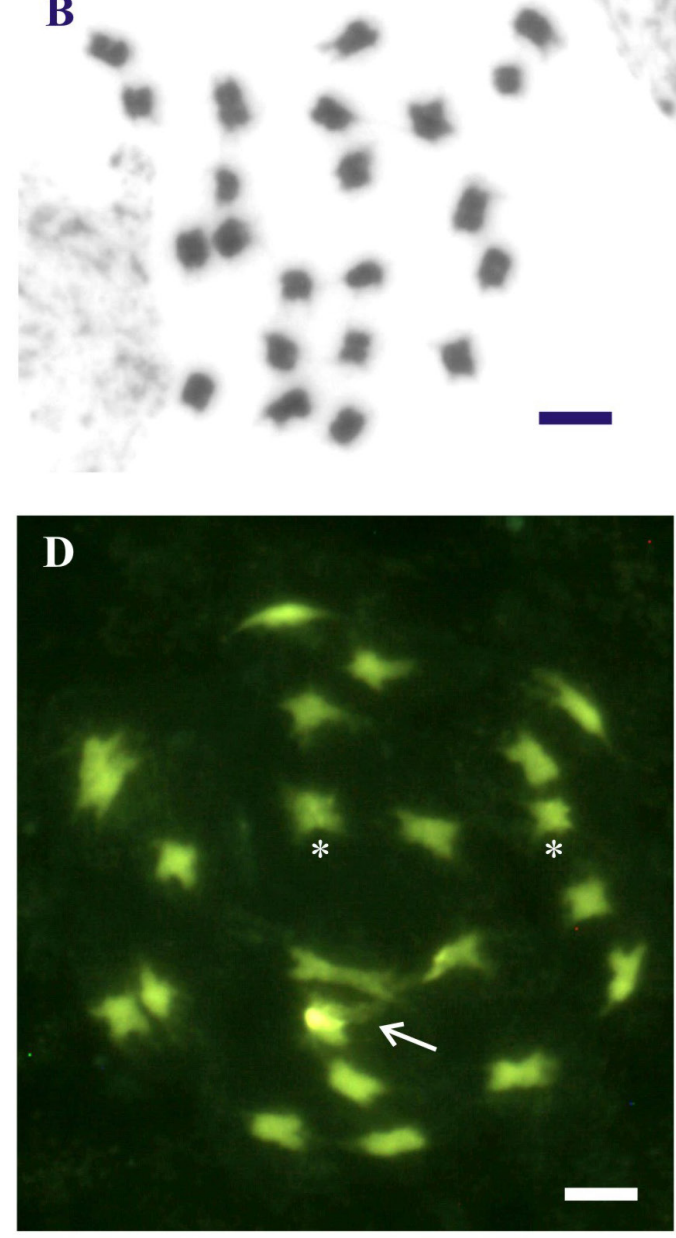

F

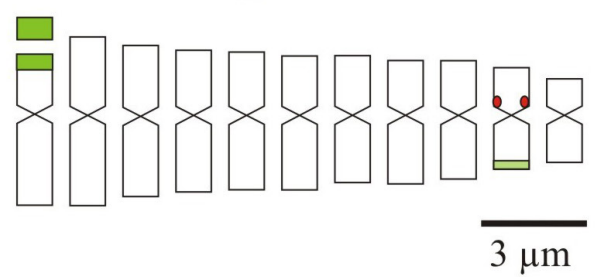

Fig. 1. Stetsonia coryne A: Histograma del contenido de ADN nuclear en picogramos por citometría de flujo, los picos M1, M2 y M3 representan los valores de 2C, 4C y 8C, de la especie estudiada. B: Metafase mitótico teñida con Giemsa. C: Bandeo cromosómico fluorescente DAPI. D: Bandeo cromosómico CMA. E: FISH usando sondas de 18-5,8-26S (verde) y 5S (rojo). Los asteriscos muestran regiones $\mathrm{CMA}^{+} / \mathrm{DAPI}^{-}$ centroméricas y las flechas regiones $\mathrm{CMA}^{+} / \mathrm{DAPl}$ /NORs. F: Idiograma, el color verde indica sitios 18-5,8$26 \mathrm{~S}$ y, el color rojo muestra regiones portadoras de $5 \mathrm{~S}$. Escalas en las fotografías: $5 \mu \mathrm{m}$. 


\section{K. Bauk et al. - ADN y patrón de genes ribosomales en el género monotípico Stetsonia}

Tabla 1. Stetsonia coryne, resultados cariotípicos. s, brazo corto; I, brazo largo; c, largo total; r, índice braquial $(r=1 / s)$; i, índice centromérico $(i=100 \mathrm{~s} / \mathrm{c}$ ). Las longitudes medias cromosómicas están expresadas en $\mu \mathrm{m}$ ( \pm desvío estándar). Abreviaturas según Levan et al. (1964).

\begin{tabular}{|ccccccc|}
\hline par & $\mathbf{s}$ & $\mathbf{I}$ & $\mathbf{c}$ & $\mathbf{r = l / s}$ & $\mathbf{i}$ & $\mathbf{n}$ \\
\hline 1 & $2,26 \pm 0,27$ & $2,58 \pm 0,05$ & 4,84 & 1,14 & 46,65 & $m$ \\
2 & $2,22 \pm 0,11$ & $2,56 \pm 0,15$ & 4,78 & 1,15 & 46,45 & $m$ \\
3 & $2,01 \pm 0,06$ & $2,30 \pm 0,15$ & 4,31 & 1,15 & 46,57 & $m$ \\
4 & $1,84 \pm 0,02$ & $2,17 \pm 0,05$ & 4,01 & 1,18 & 45,87 & $m$ \\
\hline 5 & $1,79 \pm 0,08$ & $2,11 \pm 0,10$ & 3,9 & 1,18 & 45,89 & $m$ \\
6 & $1,68 \pm 0,08$ & $2,11 \pm 0,11$ & 3,79 & 1,26 & 44,27 & $m$ \\
7 & $1,69 \pm 0,01$ & $1,90 \pm 0,05$ & 3,59 & 1,13 & 46,99 & $m$ \\
8 & $1,55 \pm 0,02$ & $1,94 \pm 0,07$ & 3,49 & 1,25 & 44,36 & $m$ \\
9 & $1,55 \pm 0,02$ & $1,81 \pm 0,09$ & 3,35 & 1,17 & 46,12 & $m$ \\
10 & $1,35 \pm 0,13$ & $1,53 \pm 0,21$ & 2,88 & 1,13 & 46,84 & $m$ \\
11 & $1,03 \pm 0,08$ & $1,33 \pm 0,12$ & 2,37 & 1,29 & 43,61 & $m$ \\
\hline
\end{tabular}

de América del norte (Opuntia) y de Cactoideae (ej. Rebutia, Mammillaria y Escobaria), (Palomino et al., 1999; Zonneveld et al., 2005; Del Angel et al., 2006; Negron -Ortiz, 2007; Las Peñas et al., 2013). En angiospermas el $90 \%$ de las especies presentan en alguno de sus tejidos somáticos células con diferentes niveles de ploidía generados por procesos de endoduplicación del genoma (D’Amato, 1984; Joubes \& Chevalier, 2000). Tal fenómeno puede variar entre los individuos de una misma especie en respuesta a diferentes condiciones ambientales (Barow, 2006; Leitch \& Leitch, 2013). Algunos autores han indicado que la endopoliploidía es beneficiosa para los cactus por poseer un tamaño pequeño del genoma (Palomino et al., 1999; Del Angel et al., 2006). Las plantas jóvenes de Stetsonia coryne mostraron una mezcla de núcleos $2 \mathrm{C}, 4 \mathrm{C}$ y $8 \mathrm{C}$, este patrón sugiere endopoliploidía. Este proceso ha sido frecuentemente observado en la subfamilia de las Opuntioideae (Negron-Ortiz, 2007; Segura et al., 2007), sin embargo en las Cactoideae solo se ha observado en algunas especies de Mammillaria (Palomino et al., 1999; Del Angel et al., 2006) y en la subfamilia Pereskioideae en Pereskia grandifolia (De Rocher et al., 1990). Stetsonia vive en regiones muy secas, en terrenos fuertemente salinos o llanuras muy áridas (Kiesling 1996, Demaio et al., 2002), este tipo de adaptaciones ecológicas se encuentran asociadas a la endopoliploidía (De Rocher et al., 1990; Negron-Ortiz, 2007; Leitch \& Leitch, 2013).

El número cromosómico es la característica cariotípicamásusada en los análisis citotaxonómicos. En Angiospermas los valores más frecuentes son $\mathrm{n}=7-20$ sin embargo, puede variar unas 160 veces (Grant, 1982). En Cactaceae, el número cromosómico básico es $\mathrm{x}=11$, este guarismo se ha confirmado para las cuatro subfamilias (e.g. Pinkava, 2002; Arakaki et al., 2007; Las Peñas, 2009; Majure et al., 2012). La especie aquí estudiada (Stetsonia coryne $2 \mathrm{n}=22$ ) coincide con los reportes para la familia.

En Cactaceae, el largo cromosómico promedio es de $2,75 \mu \mathrm{m}$, por lo que son considerados de tamaño pequeño $\mathrm{y}$, dentro de cada complemento, las diferencias de tamaño son escasas (Palomino et al., 1988; Cota \& Wallace, 1995; Cid \& Palomino, 1996; Palomino \& Heras, 2001; Briones et al., 2004, Del Angel et al., 2006; Las Peñas, 2009; Las Peñas et al., 2013), las mediciones cromosómicas obtenidas en Stetsonia coinciden con esta tendencia de homogeneidad cariotípica tanto en el tamaño, como en la morfología de los complementos cromosómicos.

En plantas el bandeo cromosómico fluorescente 
revela bandas heterocromáticas ricas en GC o AT, los resultados obtenidos en este trabajo muestran una banda en el primer par $\mathrm{m} \mathrm{CMA}^{+} / \mathrm{DAPI}^{-}$ asociado a la región organizadora nucleolar (NOR). En angiospermas es característico este tipo de bandas $\mathrm{CMA}^{+} / \mathrm{DAPI}^{-} / \mathrm{NORs}$ (Guerra, 2000), como fue identificado en $S$. coryne. La localización del gen 18-5,8-26S en Stetsonia se correlacionó siempre con las bandas de heterocromatina $\mathrm{CMA}^{+}$/ DAPI- asociadas a NORs. La localización de este gen de $\mathrm{ADNr}$ está altamente conservado en la familia (Las Peñas et al., 2009, 2013), también ha sido reportado en otras familias como Asteraceae y Solanaceae (Fregonezi et al., 2004; Ruas et al., 2005; Moreno et al., 2012). Además, se reportó la localización de $\mathrm{ADNr} 5 \mathrm{~S}$ en un par cromosómico en posición pericentromérica, coincidiendo con las demás especies de Angiospermas (Kulak et al., 2002).

Los resultados obtenidos constituirían uno de los primeros aportes al conocimiento de las características citogenéticas de la tribu Browningieae, ya que desde el punto de vista citogenético no podemos compararla con los géneros hermanos (Browningia, Jasminocereus y Neoraimondia) debido a que se desconoce los datos cromosómicos.

\section{Agradecimientos}

Los autores desean agradecer a la asistencia del Consejo Nacional de Investigaciones Científicas y Técnicas (CONICET) y la Universidad Nacional de Córdoba, ambas instalaciones de apoyo utilizados en esta investigación. Al Instituto de Investigaciones Biológicas Clemente Estable, por prestarnos los servicios para el análisis de contenido de ADN. FONCyT y SECYT-UNC brindaron soporte financiero para realizar el presente trabajo.

\section{Bibliografía}

ANDERSON, E. F. 2001. The Cactus Family. Timber Press, Portland, Oregon.

ARAKAKI, M., D. E. SOLTIS \& P. SPERANZA. 2007. New chromosome counts and evidence of polyploidy in Haageocereus and related genera in tribe Trichocereeae. Brittonia. 59: 290-297.

BANDYOPADHYAY, B. U. L. \& A. SHARMA. 2000.
The use of multivariate analysis of karyotypes to determine relationships between species of Opuntia (Cactaceae). Caryologia. 53: 121-126.

BAROW, M. 2006. Endopolyploidy in seed plants. BioEssays. 28: 271-281.

BAUK, K. \& M. L. LAS PEÑAS. 2012. Estudios citogenéticos en Stetsonia coryne (Cactaceae). Bol. Soc. Latin. Carib. Cact. Suc. Volumen 9 / No 3 Sep.Dic. 2012.

BENNETT, M. D., P. BHANDOL \& I. J. LEITCH. 2000. Nuclear DNA amounts and their modern uses: 807 new estimates. Ann. Bot. 86: 859-909.

BENNETT, M. D. \& I. J. LEITCH. 2010. Plant DNA C-values Database (release 5.0, December 2010). Available on-line: http://data.kew.org/cvalues/ (last access 15 Aug. 2011).

BERNARDELLO, G., L. STIEFKENS \& M. L. LAS PEÑAS. 2008. Karyotype studies in Grabowskia and Phrodus (Solanaceae). Pl. Syst. Evol. 275: 265-269.

BLANCO, S., M. L. LAS PEÑAS, G. BERNARDELLO \& L. STIEFKENS. 2012. Mapeo de genes ribosómicos y heterocromatina en seis especies de Lycium de Sudamérica (Solanaceae). Bol. Soc. Argent. Bot. 47: 389-399.

BOTTINI, M. C. J. 2000. Estudios multidisciplinarios en las especies patagónicas argentinas del género Berberis L. (Berberidaceae). Tesis doctoral. Universidad de Buenos Aires, Buenos Aires.

BRIONES, F., G. PALOMINO \& A. GARCIA. 2004. Chromosome analysis of Mammillaria supertexta, M. crucigera and M. haageana and their comparison with M. san-angelensis (Cactaceae). Caryologia 57: 211-218.

CID, R. \& G. PALOMINO. 1996. Cytotypes and Meiotic Behavior in Mexican Populations of Myrtillocactus geometrizans var. geometrizans (Cactaceae). Cytologia. 61: 343-348.

COSTICH, D. E., T. R. MEAGHER \& E. J. YURKOW. 1991. A rapid means of sex identification in Silene latifolia by use of flow cytometry. Pl. Mol. Biol. Rep. 9: 359-370.

COTA, J. H. \& R. S. WALLACE. 1995. Karyotypic studies in the Echinocerus (Cactaceae) and their taxonomic significance. Caryologia 48: 105-122.

D'AMATO, F. 1989. Polyploidy in cell differentiation. Caryologia 42: 183-211.

DAS, A. B., S. MOHANTY \& P. DAS. 1998 a. Interspecific variation of nuclear DNA and structural changes in meiotic and mitotic chromosome in some species of Mammillaria (Cactaceae). Caryologia 51: 289-301.

DAS, A. B., S. MOHANTY \& P. DAS. 1998 b. Interspecific variation in nuclear DNA content and chromosome analysis in Melocactus. Cytologia 63: 239-247. 
DAS, A. B., \& S. MOHANTY. 2006. Karyotype analysis and in situ nuclear DNA content in seven species of Echinopsis Zucc. of the family Cactaceae. Cytologia 71: 75-79.

DEL ANGEL, C., G. PALOMINO, A. GARCIA \& I. MENDEZ. 2006. Nuclear genome size and karyotype analysis in Mammillaria species (Cactaceae). Caryologia 59: 177-186.

DEMAIO, P., U. OLA KARLIN \& M. MEDINA. 2002. Árboles nativos del centro de Argentina, L.O.L.A. Ecosistemas Argentinos, Buenos Aires, Argentina.

DE ROCHER, E. J., K. R., HARKINS, D.W., GALBRAITH \& H. J., BOHNERT. 1990. Developmentally regulated systemic endopolyploidy in succulents with small genomes. Science 250: 99-101.

DOLEŽEL, J., J. GREILHUBER \& J. SUDA. 2007. Estimation of nuclear DNA content in plants using flow cytometry. Nat. Protoc. 2: 2233-2244.

FREGONEZI, J. N., J. M. D. TOREZAN \& A. L. L. VANZELA. 2004. A karyotypic study of three southern Brazilian Asteraceae species using fluorescence in situ hybridization with a 45S rDNA probe and C-CMA banding. Genet. Molec. Biol. 27: 223-227.

GERLACH, W. L. \& J. L. BEDBROOK. 1979. Cloning and characterization of ribosomal RNA genes from wheat and barley. Nucleic Acids Res. 7: 1869- 1885.

GUERRA, M. 2000. Patterns of heterochromatin distribution in plant chromosomes. Genet. Molec. Biol. 23: 1029-1041.

GRANT, V. 1982. Periodicities in the chromosome numbers of the angiosperms. Bot. Gaz. 143: 379389.

GREILHUBER, J. 1984. Chromosomal evidence in taxonomy. En Current concepts in plant taxonomy. Heywood H. \& Moore M. (Eds) Academic Press, London.

HERNÁNDEZ-HERNÁNDEZ, T., H. M. HERNÁNDEZ, J. A. DE-NOVA, R. PUENTE, L. E. EGUIARTE \& S. MAGALLON. 2011. Phylogenetic relationships and evolution of growth form in Cactaceae (Caryophyllales, Eudicotyledoneae). Am. J. Bot. 98:44- 61.

HUNT, D., N. TAYLOR \& G. CHARLES. 2006. The new cactus lexicon. DH Books, Milborne Port, UK.

JOUBES, J. \& C. CHEVALIER. 2000. Endoreduplication in higher plants. Pl. Mol. Biol. 43:735-745.

KIESLING, R. 1996. Stetsonia coryne (S-D) Br y R. Quеро 10: 22-25.

KIESLING, R. \& O. E. FERRARI. 2005. 100 Cactus Argentinos. Albatros ediciones, Buenos Aires, Argentina.

KIESLING, R., L. LARROCCA, J. FAÚNDEZ, D. METZING \& S. ALBESIANO. 2008. Cactaceae. In Zuolaga FO, Morrone O, Belgrano MJ (eds.).
Catálogo de las Plantas Vasculares del Cono Sur. Monogr. Syst. Bot. Missouri Bot. Gard. 107.

KULAK, S., R. HASTEROK \& J. MALUSZYNSKA. 2002. Karyotyping of Brassica amphidiploids using $5 \mathrm{~S}$ and $25 \mathrm{~S}$ rDNA as chromosome markers. Hereditas 136: 144- 150.

LAS PEÑAS, M. L. 2009. Estudios citogenéticos en Cactaceae de Argentina. Tesis Doctoral. Facultad de Ciencias Exactas, Físicas y Naturales, Universidad de Córdoba.

LAS PEÑAS, M. L., G. BERNARDELLO \& R. KIESLING. 2008. Karyotypes and fluorescent chromosome banding in Pyrrhocactus (Cactaceae). Pl. Syst. Evol. 272: 211-222.

LAS PEÑAS, M. L., J. D. URDAMPILLETA, E. R. FORNI MARTINS \& G. BERNARDELLO. 2009. Karyotypes, heterochromatin, and physical mapping of 18S-26S rDNA in Cactaceae. Cytogenet. Gen. Res. 124: 72-80.

LAS PEÑAS, M. L., G. BERNARDELLO \& R. KIESLING. 2011. Karyotype, heterochromatin, and physical mapping of 5S and 18-5.8-26S rDNA genes in Setiechinopsis mirabilis (Cactaceae). Haseltonia 16: 83-90.

LAS PEÑAS, M. L., J. D. URDAMPILLETA, B. LÓPEZCARRO, F. SANTIÑAQUE, R. KIESLING \& G. BERNARDELLO. 2013. Classical and molecular cytogenetics and DNA content in Maihuenia and Pereskia (Cactaceae). Pl. Syst. Evol. 300: 549-558.

LEITCH I. J., M. W. CHASE \& M. D. BENNETT. 1998. Phylogenetic analysis of DNA C-values provides evidence for a small ancestral genome size in flowering plants. Ann. Bot. 82 (Supplement A): 85-94.

LEITCH, I. J. \& A. R. LEITCH. 2013. Genome size diversity and evolution in land plants, en I. J. Leitch; J. Greilhuber; J. Dolezel \& J. Wendel, (eds.). Pl. gen. div. 2: 307-320.

MAJURE, L., W. JUDD, P. SOLTIS \& D. SOLTIS. 2012. Cytogeography of the Humifusa clade of Opuntia ss Mill. 1754 (Cactaceae, Opuntioideae, Opuntieae): correlations with pleistocene refugia and morphological traits in a polyploid complex. Comparative Cytogenetics 6: 53-77.

MIHALTE, L., R. E. SESTRAS, G. FESZT \& E. TAMAS. 2011. Assessment of genetic variation on four genera of Cactaceae using taxonomic, cytological and molecular markers methods. Pl. Omics J. 4: 142-148.

MORENO, N. C., L. STIEFKENS, M. L. LAS PEÑAS, A. BARTOLI, R. TORTOSA \& G. BERNARDELLO. 2012. Molecular cytogenetic studies of the "Xanthocephalum group" (Asteraceae). Pl.. Syst. Evol. 298: 1503-1514.

MURRAY, B., P. J. DE LANGE \& A. R. FERGUSON. 2005. Nuclear DNA variation, chromosome numbers 
and polyploidy in the endemic and indigenous grass flora of New Zealand. Ann. Bot. 96: 1293-1305.

NAGL, W. 1978. Endopolyploidy and polyteny in differentiation and evolution: towards an understanding of quantitative and qualitative variation of nuclear DNA in ontogeny and phylogeny. North-Holland Publishing Company, Amsterdam.

NEGRON-ORTIZ, V. 2007. Chromosome numbers, nuclear DNA content and polyploidy in Consolea (Cactaceae), an endemic cactus of the Caribbean Islands. Amer. J. Bot. 94: 1360-1370.

NYFFELER, R. 2002. Phylogenetic relationships in the cactus family (Cactaceae) based on evidence from trnK/matK and trnL-trnF sequences. Amer. J. Bot. 89: 312-326.

OHRI, D. 1998. Genome size variation and plant systematics. Ann. Bot. 82: 75-83.

PALOMINO, G., S. ZULETA \& L. SCHEINVAR. 1988. Estudios citogenéticos de dos especies y una variedad del género Nyctocereus (Cactaceae). Bol. Soc. Bot. Méx. 48: 75-79.

PALOMINO, G., J. DOLEŽEL, R. CID, I. BRUNNER, I. MÉNDEZ \& A. RUBLUO. 1999. Nuclear genome stability of Mammillaria san-angelensis (Cactaceae) regenerants induced by auxins in long term in vitro culture. Pl. Sci. 141: 191-200.

PALOMINO, G. \& H. M. HERAS, 2001. Karyotypic studies in Opuntia cochinera, O. hyptiacantha, and O. streptacantha (Cactaceae). Caryologia 54: 147154.

PERUZZI, L., I. J. LEITCH \& K. F. CAPARELLI. 2009. Chromosome diversity and evolution in Liliaceae. Ann. Bot. 103: 459-475.

PINKAVA, D. J. 2002. On the evolution of the North American Opuntioideae. En: Hunt D, N. Taylor (eds.), Studies in the Opuntioideae, pp. 78-99. Royal Botanic Gardens, Kew.
RITZ, C. M., L. MARTINS, R. MECKLENBURG, V. GOREMYKIN \& F. H. HELLWIG. 2007. The molecular phylogeny of Rebutia (Cactaceae) and its allies demonstrates the influence of paleogeography on the evolution of South American mountain cacti. Amer. J. Bot. 94: 1321-1332.

RUAS, C. F., VANZELA, A. L. L., SANTOS, M., J. N. FREGONEZI, P. M. RUAS, N. I. MATZENBACHER \& M. L. R. AGUIARPERECIN. 2005. Chromosomal organization and phylogenetic relationshipd in Hypochaeris species (Asteraceae) from Brazil. Genet. Molec. Biol. 28: 129-139.

SEGURA, S., L. SCHEINVAR, G. OLALDE, O. LEBLANC, A. MURATALLA, C. GALLEGOS \& S. FLORES. 2007. Genome sizes and ploidy levels in Mexican cactus pear species Opuntia (Tourn.) and Robustae Britton et Rose. Genet. Resour. Crop. Evol. 54: 1033-1041.

SCHUBERT, I. \& M. A. LYSAK. 2011. Interpretation of karyotype evolution should consider chromosome structural constraints. Trends Genet. 27: 207-216.

SOUZA, L. G. R., O. CROSA \& M. GUERRA. 2010. Karyological circumscription of Ipheion Rafinesque (Gilliesioideae, Alliaceae). Pl. Syst. Evol. 287: 119127.

TREVISSON, M., P. DEMAIO. 2006. Cactus de Córdoba y el centro de Argentina, L.O.L.A. Ecosistemas Argentinos, Buenos Aires, Argentina.

ZONNEVELD, B. J. M., I. J. LEITCH \& M. D. BENNETT. 2005. First nuclear DNA amounts in more than 300 angiosperms. Ann. Bot. 96: 229-244.

Recibido el 27 de junio de 2015, aceptado el 3 de febrero de 2016 . 